\title{
An Analytical Study of a Structured Overlay in the Presence of Dynamic Membership
}

\author{
Supriya Krishnamurthy ${ }^{1,3}$, Sameh El-Ansary ${ }^{1}$, Erik Aurell ${ }^{1,2}$ and Seif Haridi ${ }^{1,3}$ \\ ${ }^{1}$ Swedish Institute of Computer Science (SICS), Sweden \\ ${ }^{2}$ Department of Physics, KTH-Royal Institute of Technology, Sweden \\ ${ }^{3}$ IMIT, KTH-Royal Institute of Technology, Sweden \\ \{supriya,sameh,eaurell,seif\}@ sics.se
}

\begin{abstract}
In this paper we present an analytical study of dynamic membership (aka churn) in structured peer-to-peer networks. We use a fluid model approach to describe steadystate or transient phenomena, and apply it to the Chord system. For any rate of churn and stabilization rates, and any system size, we accurately account for the functional form of the probability of network disconnection as well as the fraction of failed or incorrect successor and finger pointers. We show how we can use these quantities to predict both the performance and consistency of lookups under churn. All theoretical predictions match simulation results. The analysis includes both features that are generic to structured overlays deploying a ring as well as Chord-specific details, and opens the door to a systematic comparative analysis of, at least, ring-based structured overlay systems under churn.
\end{abstract}

\section{INTRODUCTION}

A $\mathrm{N}$ intrinsic property of Peer-to-Peer systems is the process of never-ceasing dynamic membership. Structured Peer-to-Peer Networks (aka Distributed Hash Tables (DHTs)) have the underlying principle of arranging nodes in an overlay graph of known topology and diameter. This knowledge results in the provision of performance guarantees. However, dynamic membership continuously "corrupts/churns" the overlay graph and every DHT strives to provide a technique to "correct/maintain" the graph in the face of this perturbation.

Both theoretical and empirical studies have been conducted to analyze the performance of DHTs undergoing "churn" and simultaneously performing "maintenance". Liben-Nowell et al. [11] prove a lower bound on the maintenance rate required for a network to remain connected in the face of a given dynamic membership rate. Aspnes et al. [3] give upper and lower bounds on the number of messages needed to locate a node/data item in a DHT in the presence of node or link failures. The value of such theoretical studies is that they provide insights neutral to the details of any particular DHT. Empirical studies have also been conducted to complement these theoretical studies by showing how within the asymptotic bounds, the performance of a DHT may vary substantially

This work is funded by the European 6th FP EVERGROW project. (C)IEEE. Personal use of this material is permitted. However, permission to reprint/republish this material for advertising or promotional purposes or for creating new collective works for resale or redistribution to servers or lists, or to reuse any copyrighted component of this work in other works, must be obtained from the IEEE. depending on different DHT designs and implementation decisions. Examples include the work of: Li et al. [10], Rhea et al. [14], and Rowstron et al. [5].

In this paper, we present a fluid model of Chord [15], a specific DHT, under churn. Fluid models have been used to model data communication systems at least since the early '80ies [2], and in some sense since the work of Erlang [4]. More recently, in the context of P2P systems, it has been used to model the performance of BitTorrent [13] and the Squirrel caching system [6]. This technique has much in common with macroscopic and mesoscopic descriptions of physical and chemical phenomena (from where the term fluid has obviously been borrowed), and carries the same advantages of conciseness and computability relative to an underlying more exact description. Our analysis is directly based on the master equation approach of physical kinetics, see e.g. the text book [12], which provides a scheme for taking the various dynamical processes involved systematically into account.

The fluid model requires the notion of a state of the system. This is just a listing of the quantities one would need to know for a description of the system at a given level of detail. For Chord, we use grosso modo a level of description which requires keeping track of how many nodes there are in the system and what the state (whether correct, incorrect or failed) of each of the pointers of those nodes is. This information is not enough to draw a unique graph of network-connections because, for example, if we know that a given node has an 'incorrect' successor pointer, this still does not tell us which node it is pointing to. However, as we will see, beginning at this level of description is sufficient to keep track of most of the details of the Chord protocols. Having defined a state, the fluid model is simply a set of equations for the evolution of the probability of finding the system in this state, given the details of the dynamics. The master equation approach is useful for keeping track of the contribution of all the events which can bring about changes in the probability in a micro-instant of time i.e., evaluating all the terms in the dynamics leading to a gain or loss of this probability.

Using this formalism we investigate a probabilistic model in which peers arrive independently, distributed as a Poisson process, and life-times are exponentially distributed. While this setup is not necessary fully realistic (more realistic models can also be analyzed using master equation techniques), it is 
standard in modeling, as it typically brings out the salient features of the system with as few obscuring details from the probabilistic model as possible. We then derive the functional forms of the following: $(i)$ Chord-specific inter-node distribution properties and $(i i)$ for every outgoing pointer of a Chord node, the probability that it is in any one of its possible states. This probability is different for each of the successor and finger pointers. We then use this information to predict other quantities such as (iii) the probability that the network gets disconnected, $(i v)$ lookup consistency (number of failed lookups), and ( $v$ ) lookup performance (latency). All quantities are computed as a function of the parameters involved and all results are verified by simulations.

\section{RELATED WORK}

Closest in spirit to our work is the informal derivation in the original Chord paper [15] of the average number of timeouts encountered by a lookup. This quantity was approximated there by the product of the average number of fingers used in a lookup times the probability that a given finger points to a departed node. Our methodology not only allows us to derive the latter quantity systematically but also demonstrates how this probability depends on which finger (or successor) is involved. Further we are able to derive a precise relation relating this probability to lookup performance and consistency accurately at any value of the system parameters.

In the works of Aberer et al. [1] and Wang et al. [16], DHTs are analyzed under churn and the results are compared with simulations. These analyses can also be classified as fluid models. However the main parameter is the probability that a random selected entry of a routing table is stale. In our analysis, we determine this quantity from system details and churn rates.

A brief announcement of the results presented in this paper, has appeared earlier in [8].

\section{OUR IMPLEMENTATION OF CHORD}

The Chord Ring. The general philosophy of DHTs is to map a set of data items onto a set of nodes where the insertion and lookup of items is done using the unique keys that the items are given. Chord's realization of that philosophy is as follows. Peers and data items are given unique keys (usually obtained by a cryptographic hash of unique attribute like the IP address or public key for nodes, and filename or checksum for items) drawn from a circular key space of size $\mathcal{K}$. The Chord system dictates that the right place for storing an item is at the first alive node whose key succeeds the key of the item. Since we refer to nodes and items by their keys, the insertion and lookup of items becomes a matter of locating the right "successor" of a key. All nodes have successor and predecessor pointers. For $N$ nodes, using only the successor pointers to lookup items requires $\frac{1}{2} N$ hops on average.

Fingers. To reduce the average lookup path length, nodes keep $\mathcal{M}=\log _{2} \mathcal{K}$ pointers known as the "fingers". Using these fingers, a node can retrieve any key in $O(\log N)$ hops. The fingers of a node $n$ (where $n \in 0 \cdots \mathcal{K}-1$ ) point to exponentially increasing distances of keys away from $n$. That is, $\forall i \in 1 . . \mathcal{M}, n$ points to a node whose key is equal to $n+2^{i-1}$. We denote that key by $n$. fin $n_{i}$.start. However, for a certain $i$, there might not be a node in the network whose key is equal to $n+2^{i-1}$. Therefore, $n$ points to the first successor of $n+2^{i-1}$ which we denote by $n$. fin $_{i}$. node.

The Successor List Moreover, each node keeps a list of the $\mathcal{S}=O(\log (N))$ immediate successors as backups for its first successor. We use the notation n.s to refer to this list and $n . s_{i}$ to refer to the $i^{t h}$ element in the list. Finally we use the notation n.p to refer to the predecessor.

Stabilization, Churn \& Steady State. To keep the pointers up-to-date in the presence of churn, each node performs periodic stabilization of its successors and fingers. In our analysis, we define $\lambda_{j}$ as the rate of joins per node, $\lambda_{f}$ the rate of failures per node and $\lambda_{s}$ the rate of stabilizations per node. The fraction of stabilizations which act on the successors is $\alpha$, such that the rate of successor stabilizations is $\alpha \lambda_{s}$, and the rate of finger stabilizations is $(1-\alpha) \lambda_{s}$. In all that follows, we impose the steady state condition $\lambda_{j}=\lambda_{f}$ unless otherwise stated. Further it is useful to define $r \equiv \frac{\lambda_{s}}{\lambda_{f}}$ which is the relevant ratio on which all the quantities we are interested in will depend, e.g, $r=50$ means that a join/fail event takes place every half an hour for a stabilization which takes place once every 36 seconds. Throughout the paper we will use the terms $\lambda_{j} N \Delta t, \lambda_{f} N \Delta t, \alpha \lambda_{s} N \Delta t$ and $(1-\alpha) \lambda_{s} N \Delta t$ to denote the respective probabilities that a join, failure, a successor stabilization, or a finger stabilization take place anywhere on the ring during a micro period of time of length $\Delta t$.

Parameters. The parameters of the problem are hence: $\mathcal{K}$, $N, \alpha$ and $r$. All relevant measurable quantities should be entirely expressible in terms of these parameters.

Simulation Since we are collecting statistics like the probability of a particular finger pointer to be wrong, we need to repeat each experiment 100 times before obtaining wellaveraged results. The total simulation sequential real time for obtaining the results of this paper was about 1800 hours that was parallelized on a cluster of 14 nodes where we had $N=1000, \mathcal{K}=2^{20}, \mathcal{S}=6,200 \leq r \leq 2000$ and $0.25 \leq \alpha \leq 0.75$.

While the main outlines of the chord protocol are provided by its authors in [15], an exact analysis necessitates the provision of a deeper level of detail and adopted assumptions which we provide in the following subsections.

\section{A. Joins, Failures \& Ring Stabilization}

Initialization. Initially, a node knows its key and at least one node with key $c$ that already exists in the network and is alive. The knowledge of such a node is assumed to be acquired through some out-of-band method. The predecessor $p$, successors $\left(s_{1 . . S}\right)$ and fingers $\left(f_{i n} \ldots \mathcal{M}\right.$.node) are all assigned to nil.

Joins (Fig. 10. A new node $n$ joins by looking up its successor using the initial random contact node $c$. It also starts its first stabilization of the successors and initializes its fingers.

Stabilization of Successors (Fig. 1). The function fixSuccessors is triggered periodically with rate $\alpha \lambda_{s}$. A node $n$ tells its first alive successor $y$ that it believes itself to be $y$ 's 




Fig. 1

JOINS AND RING STABILIZATION ALGORITHMS.

predecessor and expects as an answer $y$ 's predecessor $y . p$ and successors $y . s$. The response of $y$ can lead to three actions:

Case A. Some node exists between $n$ and $y$ (i.e., $n$ 's belief is wrong), so $n$ prepends $y . p$ to its successor list as a first successor and retries fixSuccessors.

Case B. $y$ confirms $n$ 's belief and informs $n$ of $y$ 's old predecessor $y . p$. Therefore $n$ considers $y . p$ as an alternative/initial predecessor for $n$. Finally, $n$ reconciles its successor list with y.s.

Case C. $y$ agrees that $n$ is its predecessor and the only task of $n$ is to update its successor list by reconciling it with y.s.

By calling iThinkIamYourPred (Fig. 1), some node $x$ informs $n$ that it believes itself to be $n$ 's predecessor. If $n$ 's predecessor $p$ is not alive or nil, then $n$ accepts $x$ as a predecessor and informs $x$ about this agreement by returning $x$. Alternatively, if $n$ 's predecessor $p$ is alive (discovering that will be explained shortly in section III-C, then there are two possibilities: The first is that $x$ is in the region between $n$ and its current predecessor $p$, therefore $n$ should accept $x$ as a new predecessor and inform $x$ about its old predecessor. The second is that $p$ is already pointing to $x$ so the state is correct at both parties and $n$ confirms that to $x$ by informing it that $x$ is the predecessor of $n$. In all cases the function returns a predecessor and a successor list.

The function firstAliveSuccessor (Fig. 1) iterates through the successor list. In each iteration, if the first successor $s_{1}$ is alive, it is returned. Otherwise, the dead successor is dropped from the list and nil is appended to the end of the list. If the first successor is nil this means that all immediate successors are dead and that the ring is disconnected.

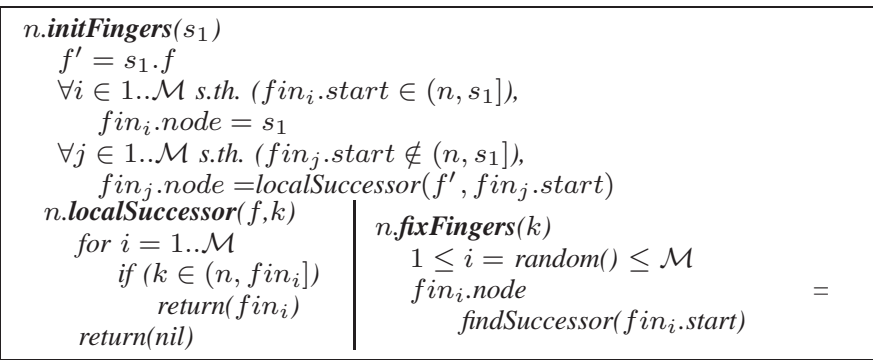

Fig. 2

INITIALIZATION AND STABILIZATION OF FINGERS.

\section{B. Lookups and Stabilization of Fingers}

Stabilization of Fingers (Fig. 2). Stabilization of fingers occurs at a rate $(1-\alpha) \lambda_{s}$. Each time the fixFingers function is triggered, a random finger fin $_{i}$ is chosen and a lookup for $f_{i n}$.start is performed and the result is used to update fin $_{i}$. node.

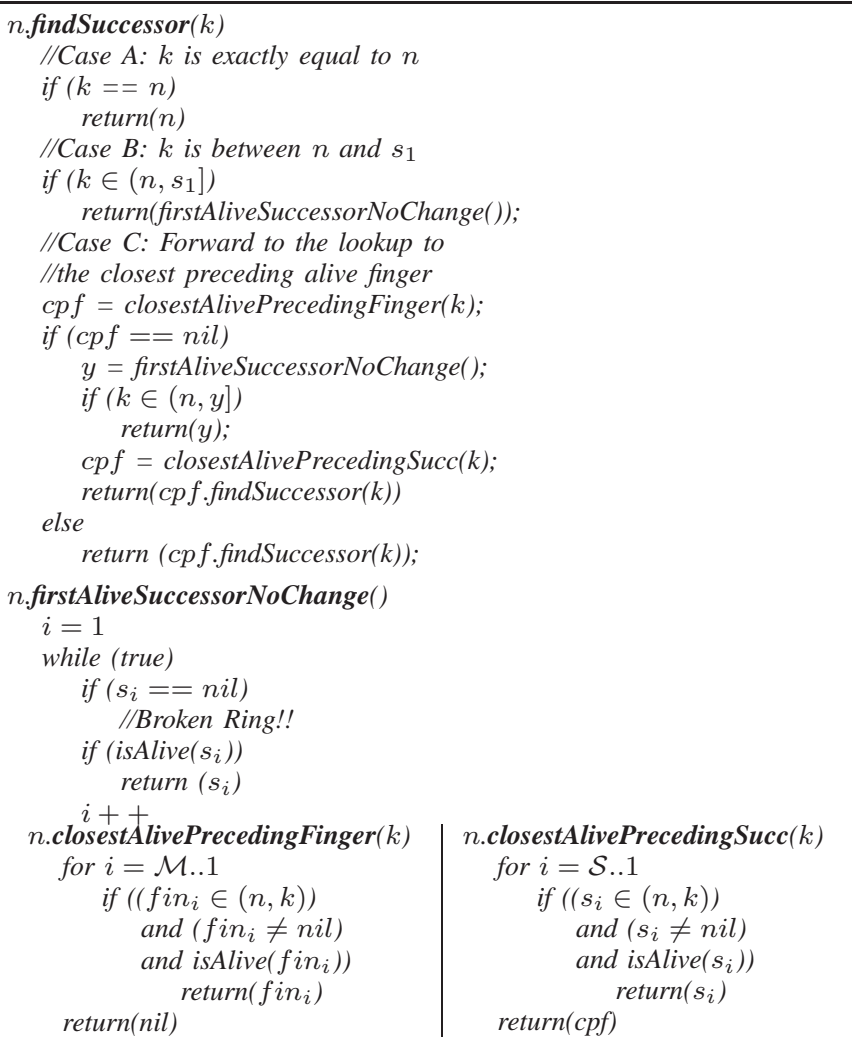

Fig. 3

THE LOOKUP ALGORITHM.

Initialization of Fingers (Fig.2). After having initialized its first successor $s_{1}$, a node $n$ sets all fingers with starts between $n$ and $s_{1}$ to $s_{1}$. The rest of the fingers are initialized by taking a copy of the finger table of $s_{1}$ and finding an approximate successor to every finger from that finger table.

Lookups (Fig. 3). A lookup operation is a fundamental operation that is used to find the successor of a key. It is used by many other routines and its performance and consistency 
are the main quantities of interest in the evaluation of any DHT. A node $n$ looking up the successor of $k$ runs the findSuccessor algorithm which can lead to the following cases:

Case $A$. If $k$ is equal to $n$ then $n$ is trivially the successor of $k$.

Case B. If $k \in\left(n, s_{1}\right]$ then $n$ has found the successor of $k$, but it could be that $s_{1}$ has failed and $n$ has not yet discovered this. However, entries in the successor list can act as backups for the first successor. Therefore, the first alive successor of $n$ is the successor of $k$. Note that, in this case, while we try to find the first alive successor, we do not change the entries in the successor list. This is mainly because, to simplify the analysis, we want the successor list to be changed at a fixed rate rate $\alpha \lambda_{s}$ only by the fixSuccessors function.

Case $C$. The lookup should be forwarded to a node closer to $k$, namely the closest alive finger preceding $k$ in $n$ 's finger table. The call to the function closestAlivePrecedingFinger returns such a node if possible and the lookup is forwarded to it. However, it could be the case that all alive preceding fingers to $k$ are dead. In that case, we need to use the successor list as a last resort for the lookup. Therefore, we locate the first alive successor $y$ and if $k \in(n, y]$ then $y$ is the successor of $k$. Otherwise, we locate the closest alive preceding successor to $k$ and forward the lookup to it.

\section{Failures}

Throughout the code we use the call is Alive and isNotAlive. A simple interpretation of those routines would be to equate them to a performance of a ping. However, a correct implementation for them is that they are discovered by performing the operation required. For instance, a call to firstAliveSuccesor in Fig. 1 is performed to retrieve a node $y$ and then call $y . i$ ThinkIamYourPred, so alternatively the first alive successor could be discovered by iterating on the successor list and calling iThinkIamYourPred.

\section{The ANALYsis}

\section{A. Distributional Properties of Inter-Node Distances}

In this section we will assume that all keys are populated by peers with independent and equal probability, and, furthermore, that this probability does not change with time. The first condition is a natural consequence of peers joining and leaving/failing independently. The last condition, on the other hand, does not hold strictly since the number of peers present under churn is a fluctuating quantity, Nevertheless, it can be expected to hold to good accuracy in sufficiently large systems. A detailed analysis along these lines will be given elsewhere.

Definition 4.1: Given two keys $u, v \in\{0 \ldots \mathcal{K}-1\}$, the "distance" between them is $u-v$ (with modulo- $\mathcal{K}$ arithmetic). We interchangeably say that $u$ and $v$ form an "interval" of length $u-v$. Hence the number of keys inside an interval of length $\ell$ is $\ell-1$ keys.

Property 4.1: The probability $P(x)$ of finding an interval of length $x$ is: $P(x)=\rho^{x-1}(1-\rho)$ where $\rho=\frac{\mathcal{K}-N}{\mathcal{K}}$.

Under the stated conditions, each key will be populated with the same probability $\frac{N}{\mathcal{K}}=1-\rho$, for $N<<K$. An interval

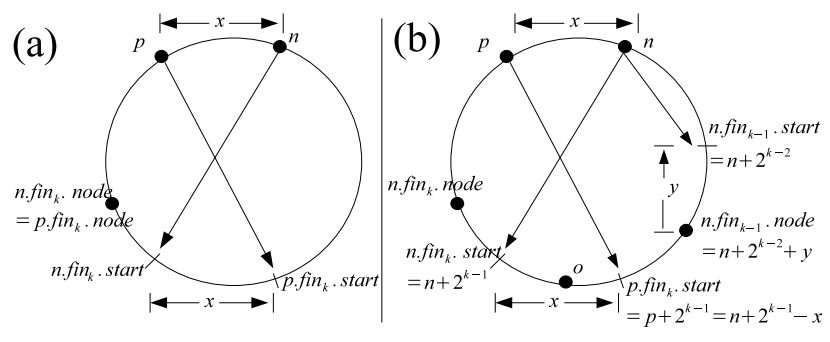

Fig. 4

(A) CASE WHEN $n$ AND $p$ HAVE THE SAME VALUE OF f $i n_{k}$.node. (B) CASE WHERE A NEWLY JOINED NODE $p$ COPIES THE $k^{\text {th }}$ ENTRY OF ITS SUCCESSOR NODE $n$ AS THE BEST APPROXIMATION FOR ITS OWN $k^{t h}$ ENTRY (BY THE JOIN PROTOCOL). IN THIS CASE, THERE COULD BE A NODE $o$ WHICH IS THE 'CORRECT' ENTRY FOR $p$. fin $_{k}$. node. HOWEVER, SINCE $p$ IS NEWLY JOINED, THE ONLY INFORMATION IT HAS ACCESS TO IS THE FINGER TABLE OF $n$.

of length $x$ then involves $x-1$ consecutive unpopulated keys, and then one populated key, which explains the formula.

We now derive some properties of this distribution which will be used in the ensuing analysis.

Property 4.2: For any two keys $u$ and $v$, where $v=u+x$, let $b_{i}$ be the probability that the first node encountered in between these two keys is at $u+i$ (where $0 \leq i<x$ ). Then $b_{i} \equiv \rho^{i}(1-\rho)$. The probability that there is definitely at least one node between $u$ and $v$ is: $a(x) \equiv 1-\rho^{x}$. Hence the conditional probability that the first node is at distance $i$ given that there is at least one node in the interval is $b c(i, x) \equiv$ $b(i) / a(x)$.

Property 4.3: The probability that a node and at least one of its immediate predecessors share the same $k^{\text {th }}$ finger is $p_{1}(k) \equiv \frac{\rho}{1+\rho}\left(1-\rho^{2^{k}-2}\right)$. The explanation for this property goes as follows. If the distance between node $n$ and its predecessor $p$ is $x$, the distance between $n$. fin $_{k}$.start and p. fin $_{k}$. start is also $x$ (see Fig. 4 (a)). If there is no node in

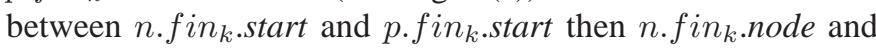
p. fin $_{k}$.node will share the same value. From Property 4.1, the probability that the distance between $n$ and $p$ is $x$ is $\rho^{x-1}(1-\rho)$. However, $x$ has to be less than $2^{k-1}$, otherwise p. fin $_{k}$.node will be equal to $n$. The probability that no

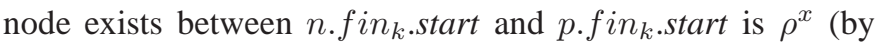
Property 4.2). Therefore the probability that the $n$. fin $n_{k}$.node and $p$. in $_{k}$.node share the same value is: $\sum_{x=1}^{2^{k-1}-1} \rho^{x-1}(1-$ $\rho) \rho^{x}=\frac{\rho}{1+\rho}\left(1-\rho^{2^{k}-2}\right)$. It is straightforward (though tedious) to derive similar expressions for $p_{2}(k)$ the probability that a node and at least two of its immediate predecessors share the same $k^{\text {th }}$ finger, $p_{3}(k)$ and so on.

Property 4.4: We can similarly assess the probability that the join protocol (see Section III-B) results in further replication of the $k^{t h}$ pointer. Let us define the probability $p_{\text {join }}(i, k)$ as the probability that a newly joined node, chooses the $i^{\text {th }}$ entry of its successor's finger table for its own $k^{\text {th }}$ entry. Note that this is unambiguous even in the case that the successor's $i^{t h}$ entry is repeated. All we are asking is, when is the $k^{t h}$ entry of the new joinee the same as the $i^{\text {th }}$ entry of the successor? 


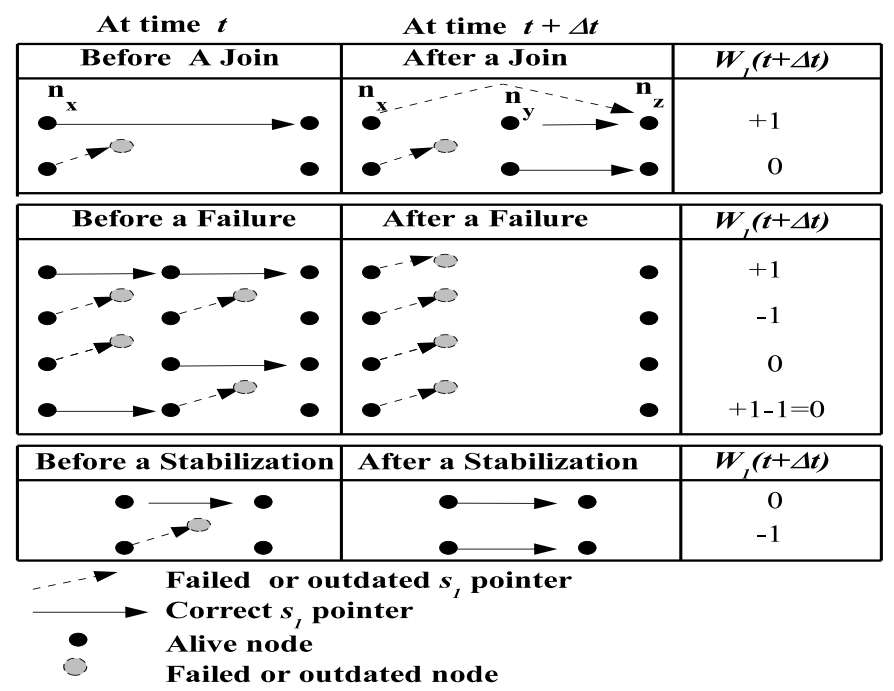

Fig. 5

CHANGES IN $W_{1}$, THE NUMBER OF WRONG (FAILED OR OUTDATED) $s_{1}$ POINTERS, DUE TO JOINS, FAILURES AND STABILIZATIONS.

Clearly $i \leq k$. In fact for the larger fingers, we only need to consider $p_{\text {join }}(k, k)$, since $p_{\text {join }}(i, k) \sim 0$ for $i<k$. Using the interval distribution we find, for large $k, p_{\text {join }}(k, k) \sim \rho(1-$ $\left.\rho^{2^{k-2}-2}\right)+(1-\rho)\left(1-\rho^{2^{k-2}-2}\right)-(1-\rho) \rho\left(2^{k-2}-2\right) \rho^{2^{k-2}-3}$. This function goes to 1 for large $k$.

We can also analogously compute $p_{\text {join }}(i, k)$ for any $i$. The only trick here is to estimate the probability that starting from $i$, the last distinct entry of $n$ 's finger table does not give $p$ a better choice for its $k_{t h}$ entry. This can again readily be computed using property 4.2 , but we do not do the computation here since for our purposes $p_{\text {join }}(k, k)$ suffices.

\section{B. Successor Pointers}

We now turn to estimating various quantities of interest for Chord. In all that follows we will evaluate various average quantities, as a function of the parameters. To do this we need to understand how the dynamical evolution of the system affects these quantities.

In the case of Chord, we only need to consider one of three kinds of events happening at any micro-instant: a join, a failure or a stabilization. One assumption made in the following is that such a micro-instant of time exists, or in other words, that we can divide time till we have an interval small enough that in this interval, only one of these three processes occurs anywhere in the system. Implicit in this is the assumption that a stabilization (either of successors or fingers) is done faster than the time-scales over which joins and fails occur.

Another aspect of this system which simplifies analysis is that successor pointers of adjacent nodes are independent of each other. That is, the state of the first successor pointer of a given node does not affect the state of the first successor pointer of either its predecessor or its successor. The same logic also works for the state of the second successor pointers of adjacent nodes and so on. On the other hand, the state of
TABLE I

GAIN AND LOSS TERMS FOR $W_{1}(r, \alpha)$ : THE NUMBER OF WRONG FIRST SUCCESSORS AS A FUNCTION OF $r$ AND $\alpha$.

\begin{tabular}{|l|l|}
\hline Change in $W_{1}(r, \alpha)$ & Probability of Occurrence \\
$W_{1}(t+\Delta t)=W_{1}(t)+1$ & $c_{1.1}=\left(\lambda_{j} N \Delta t\right)\left(1-w_{1}\right)$ \\
$W_{1}(t+\Delta t)=W_{1}(t)+1$ & $c_{1.2}=\lambda_{f} N\left(1-w_{1}\right)^{2} \Delta t$ \\
$W_{1}(t+\Delta t)=W_{1}(t)-1$ & $c_{1.3}=\lambda_{f} N w_{1}^{2} \Delta t$ \\
$W_{1}(t+\Delta t)=W_{1}(t)-1$ & $c_{1.4}=\alpha \lambda_{s} N w_{1} \Delta t$ \\
$W_{1}(t+\Delta t)=W_{1}(t)$ & $1-\left(c_{1.1}+c_{1.2}+c_{1.3}+c_{1.4}\right)$ \\
\hline
\end{tabular}

the second successor pointer of a node is clearly related to the state of its first successor pointer as well the state of the first successor pointer of the successor. This is taken into account in the analysis of second and higher successor pointers. In characterizing the states of higher successors, we look for the leading order behavior in terms of the parameter $r$.

Consider first the successor pointers. Let $w_{k}(r, \alpha)$ denote the fraction of nodes having a wrong $k^{\text {th }}$ successor pointer and $d_{k}(r, \alpha)$ the fraction of nodes having a failed successor pointer. Also, let $W_{k}(r, \alpha)$ be the number of nodes having a wrong $k^{\text {th }}$ successor pointer and $D_{k}(r, \alpha)$ the number of nodes having a failed successor pointer. A failed pointer is one which points to a departed node while a wrong pointer points either to an incorrect node (alive but not correct) or a dead one. As we will see, both these quantities play a role in predicting lookup consistency and lookup length.

By the protocol for stabilizing successors in Chord, a node periodically contacts its first successor, possibly correcting it and reconciling with its successor list. Therefore, the number of wrong $k^{t h}$ successor pointers are not independent quantities but depend on the number of wrong first successor pointers.

We write an equation for $W_{1}(r, \alpha)$ by accounting for all the events that can change it in a micro event of time $\Delta t$. An illustration of the different cases in which changes in $W_{1}$ take place due to joins, failures and stabilizations is provided in Fig. 5 In some cases $W_{1}$ increases/decreases while in others it stays unchanged. For each increase/decrease, Table \provides the corresponding probabilities.

By our implementation of the join protocol, a new node $n_{y}$, joining between two nodes $n_{x}$ and $n_{z}$, always has a correct $s_{1}$ pointer after the join. However the state of $n_{x} . s_{1}$ before the join makes a difference. If $n_{x} . s_{1}$ was correct (pointing to $n_{z}$ ) before the join, then after the join it will be wrong and therefore $W_{1}$ increases by 1 . If $n_{x} . s_{1}$ was wrong before the join, then it will remain wrong after the join and $W_{1}$ is unaffected. Thus, we need to account for the former case only. The probability that $n_{x} . s_{1}$ is correct is $1-w_{1}$ and term $c_{1.1}$ follows from this.

For failures, we have 4 cases. To illustrate them we use nodes $n_{x}, n_{y}, n_{z}$ and assume that $n_{y}$ is going to fail. First, if both $n_{x} \cdot s_{1}$ and $n_{y} \cdot s_{1}$ were correct, then the failure of $n_{y}$ will make $n_{x} . s_{1}$ wrong and hence $W_{1}$ increases by 1 . Second, if $n_{x} . s_{1}$ and $n_{y} . s_{1}$ were both wrong, then the failure of $n_{y}$ will decrease $W_{1}$ by one, since one wrong pointer disappears. Third, if $n_{x} . s_{1}$ was wrong and $n_{y} . s_{1}$ was correct, then $W_{1}$ is unaffected. Fourth, if $n_{x} . s_{1}$ was correct and $n_{y} \cdot s_{1}$ was wrong, then the wrong pointer of $n_{y}$ disappears and $n_{x} . s_{1}$ becomes wrong, therefore $W_{1}$ is unaffected. For the first case 




Fig. 6

THEORY AND SIMULATION FOR THE PROBABILITY OF WRONG $1^{\text {st }}$ SUCCESSOR $w_{1}(r, \alpha)$ AND FAILED $1^{\text {st }}$ SUCCESSOR $d_{1}(r, \alpha)$.

to happen, we need to pick two nodes with correct pointers, the probability of this is $\left(1-w_{1}\right)^{2}$. For the second case to happen, we need to pick two nodes with wrong pointers, the probability of this is $w_{1}^{2}$. From these probabilities follow the terms $c_{1.2}$ and $c_{1.3}$.

Finally, a successor stabilization does not affect $W_{1}$, unless the stabilizing node had a wrong pointer. The probability of picking such a node is $w_{1}$. From this follows the term $c_{1.4}$.

Hence the equation for $W_{1}(r, \alpha)$ is:

$$
\frac{d W_{1}}{N d t}=\lambda_{j}\left(1-w_{1}\right)+\lambda_{f}\left(1-w_{1}\right)^{2}-\lambda_{f} w_{1}^{2}-\alpha \lambda_{s} w_{1}
$$

Solving for $w_{1}$ in the steady state and putting $\lambda_{j}=\lambda_{f}$, we get:

$$
w_{1}(r, \alpha)=\frac{2}{3+r \alpha} \approx \frac{2}{r \alpha}
$$

This expression matches well with the simulation results as shown in Fig. 6 $d_{1}(r, \alpha)$ is then $\approx \frac{1}{2} w_{1}(r, \alpha)$ since when $\lambda_{j}=\lambda_{f}$, about half the number of wrong pointers are incorrect and about half point to dead nodes. Thus $d_{1}(r, \alpha) \approx \frac{1}{r \alpha}$ which also matches well the simulations as shown in Fig. 6

The fraction of wrong second successors can be estimated in an analogous manner. Consider, for a node $n$, the possible states of the successor, n.s. $*\left(n . s_{1}\right) . s_{1}$, and the second successor, n.s. $s_{2}$. In a fully correct state, $*\left(n . s_{1}\right) . s_{1}$ and $n . s_{2}$ of course point to the same node. If in such a state either $n . s_{1}$ or $*\left(n . s_{1}\right) . s_{1}$ becomes incorrect through the action of a join or a failure, then $n . s_{2}$ is also incorrect. On the other hand, n.s. cannot be corrected by the stabilization protocol unless both $n . s_{1}$ and $*\left(n . s_{1}\right) . s_{1}$ are both already corrected. Hence, $n . s_{2}$ is wrong if either $n . s_{1}$ or $*\left(n . s_{1}\right) . s_{1}$ are wrong, and also if both $n . s_{1}$ and $*\left(n . s_{1}\right) . s_{1}$ are correct, but $n . s_{2}$ has not yet been corrected. If the number of such non-stabilized configurations is $N_{2}$ and the fraction is $n_{2}$, we have

$$
w_{2}=2 w_{1}-w_{1}^{2}+n_{2}
$$

To estimate $n_{2}$ we consider how these configurations might be gained or lost. The gain term arises from stabilizations of configurations where $n . s_{1}$ is correct but $*\left(n . s_{1}\right) . s_{1}$ is wrong. A stabilization performed by node $n . s_{1}$ then results in the gain of a $N_{2}$ configuration. On the other hand, non-stabilized configurations are lost either by a stabilization performed by node $n$ (when it gets the correct successor list from its successor and hence corrects $n . s_{2}$ ), or by corrupting either $n . s_{1}$ or $*\left(n . s_{1}\right) . s_{1}$ (by a join or failure). The latter possibility gives terms of order $\frac{1}{r^{2}}$ and we can ignore it in the limit that stabilizations happens on a much faster time scale than joins and failures (i.e., $r$ much larger than unity). The equation for $\mathrm{N}_{2}$ is hence

$$
\frac{d N_{2}}{d t} \approx \alpha \lambda_{s} w_{1}\left(1-w_{1}\right)-\alpha \lambda_{s} n_{2}
$$

which implies $n_{2} \approx w_{1}$ to order $\frac{1}{r}$. Thus, we have $w_{2} \approx \frac{6}{r}$.

For higher successors we reason similarly by considering the state of the $k-1^{s t}$ successor pointer of node $n$, the successor pointer of the $k-1^{s t}$ successor, and the $k^{t h}$ successor pointer of node $n$. We can write a recursion equation for $w_{k}$ the fraction of nodes with wrong $k^{t h}$ successor pointer

$$
w_{k}=w_{1}+w_{k-1}-w_{k-1} w_{1}+n_{k}
$$

where $n_{k}$ is the density of configurations where the $k-1^{s t}$ successor pointer of node $n$ and the first successor pointer of the $k-1^{\text {st }}$ successor are both correct, but this information has not yet been used to correct the $k^{\text {th }}$ successor pointer of node $n$. If node $n$ does not as yet have the correct information about its $k^{\text {th }}$ successor, that means that either all the nodes in between $n$ and its $k-1^{\text {st }}$ successor have the correct information but node $n$ has not as yet stabilized, or that the stabilization has propagated back from the $k-1^{\text {st }}$ successor to some node in between but not as yet to $n . s_{1}$. To elaborate on this further, there is the case where the second successor pointer of the $k-2^{\text {nd }}$ successor has not been corrected, then the case where this has been done, but the third successor pointer of the $k-3^{\text {rd }}$ successor has not been corrected, and so on. Each of these is analogous to $n_{2}$ and each occurs with density $\left(1-w_{k-1}\right) w_{1}$, if joins and failures are neglected compared to stabilizations. Hence, if to leading order in $\frac{1}{r}$ we have $w_{k} \sim \frac{c_{k}}{\alpha r}$, then

$$
c_{k}=c_{k-1}+k c_{1}
$$

which leads to

$$
w_{k} \approx \frac{k(k+1)}{\alpha r}
$$

. We note that this expression obviously depends on the details of the stabilization scheme, and is in principle only valid up to $k \sim \sqrt{r}$. As shown in Fig. 7, the agreement between theory and simulation is still however quite reasonable at $k=5$ and $r=100$.

\section{Break-up (Network Disconnection) Probability}

We demonstrate below, how calculating $d_{k}(r, \alpha)$ : the fraction of nodes with dead $k^{\text {th }}$ pointers, helps in estimating the probability that the network gets disconnected for any value of $r$ and $\alpha$. Let $P_{b u}(n, r, \alpha)$ be the probability that $n$ consecutive nodes fail. If $n=\mathcal{S}$, the length of the successor list, then 


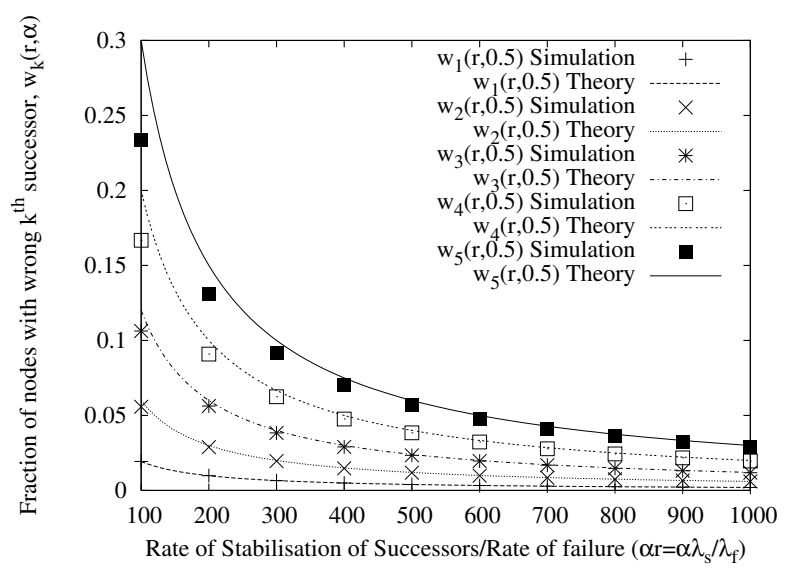

Fig. 7

THEORY AND SIMULATION FOR THE PROBABILITY OF A WRONG $k^{t h}$ SUCCESSOR $w_{k}(r, \alpha)$.

TABLE II

GAIN AND LOSS TERMS FOR $N_{b u}(2, r, \alpha)$ : THE NUMBER OF NODES WITH DEAD FIRST and SECOND SUCCESSORS.

\begin{tabular}{|l|l|}
\hline Change in $N_{b u}(r, \alpha)$ & Probability of Occurrence \\
$N_{b u}(t+\Delta t)=N_{b u}(t)+1$ & $c_{2.1}=\left(\lambda_{f} N \Delta t\right) d_{1}(r, \alpha)$ \\
$N_{b u}(t+\Delta t)=N_{b u}(t)+1$ & $c_{2.2}=\lambda_{f} N \Delta t\left(1-d_{1}\right) d_{2}$ \\
$N_{b u}(t+\Delta t)=N_{b u}(t)-1$ & $c_{2.3}=\alpha \lambda_{s} N \Delta t P_{b u}(2, r, \alpha)$ \\
$N_{b u}(t+\Delta t)=N_{b u}(t)$ & $1-\left(c_{2.1}+c_{2.2}+c_{2.3}\right)$ \\
\hline
\end{tabular}

clearly the node whose successor list this is, gets disconnected from the network and the network breaks up. For the range of $r$ considered in Fig. 6, $P_{b u}(\mathcal{S}, r, \alpha) \sim 0$. However should we go lower, this starts becoming finite. The master equation analysis introduced here can be used to estimate $P_{b u}(n, r, \alpha)$ for any $1 \leq n \leq \mathcal{S}$. We indicate how this might be done by first considering the case $n=2$. Let $N_{b u}(2, r, \alpha)$ be the number of configurations in which a node has both $s_{1}$ and $s_{2}$ dead and $P_{b u}(2, r, \alpha)$ be the fraction of such configurations. Table I indicates how this is estimated within the present framework.

A join event does not affect this probability in any way. So we only need to consider the effect of failures or stabilization events. The term $c_{2.1}$ accounts for the situation when the first successor of a node is dead (which happens with probability $d_{1}(r, \alpha)$ as explained above). A failure event can then kill its second successor as well and this happens with probability $c_{2.1}$. The second term is the situation that the first successor is alive (with probability $1-d_{1}$ ) but the second successor is dead (with probability $d_{2}$ ). The logic used to estimate $d_{2}$ (or $d_{k}$ in general) is very similar to the reasoning we used to estimate the $w_{k}$ 's. So we have

$$
d_{k}=d_{1}+(k-1) d_{1}=k d_{1}
$$

Thus the $k^{t h}$ successor of a node is dead if the $k-1^{\text {st }}$ successor's successor is dead, or the $k-1^{\text {st }}$ successor's successor is not dead but the intermediate nodes think it is because they haven't stabilized. Hence $d_{2} \sim 2 / \alpha r$. This estimate for $d_{2}$ matches the simulation results very well, as shown in Fig. 8

Coming back to counting the gain and loss terms for



THEORY AND SIMULATION FOR THE PROBABILITY OF FAILURE OF THE $2^{\text {nd }}$ SUCCESSOR, $d_{2}(r, \alpha)$.

$N_{b u}(2, r, \alpha)$, a stabilization event reduces the number of such configurations by one, if the node doing the stabilization had such a configuration to begin with.

Solving the equation for $N_{b u}(2, r, \alpha)$, one hence obtains that $P_{b u}(2, r, \alpha) \sim 3 /(\alpha r)^{2}$. As Fig. 9 shows, this is a precise estimate.

We can similarly estimate the probabilities for three consecutive nodes failing, etc, and hence also the general disconnection probability $P_{b u}(\mathcal{S}, r, \alpha)$. In fact $P_{b u}(\mathcal{S}, r, \alpha)$ may be written in terms of the $d_{k}(r, \alpha)$ as:

$$
P_{b u}(\mathcal{S})=(\mathcal{S}-1) ! \frac{\sum_{1}^{\mathcal{S}} d_{i}(r, \alpha)}{(\alpha r)^{\mathcal{S}-1}}
$$

The logic behind this equation is similar to that used for solving for $P_{b u}(2)$, namely that for $\mathcal{S}$ consecutive nodes to fail, any $\mathcal{S}-1$ of the $\mathcal{S}$ nodes should have failed first, and then a failure event kills the remaining node. (8) is readily solved by substituting the values of the $d_{k}$ 's to get

$$
P_{b u}(\mathcal{S})=\frac{(\mathcal{S}+1) !}{2(\alpha r)^{\mathcal{S}}}
$$

As mentioned above this is again correct only to leading order. Namely there will be correction terms of the order $r^{\mathcal{S}+1}$ which we haven't computed at this level of approximation. The Master Equation formalism thus affords the possibility of making a precise prediction for when the system runs the danger of getting disconnected, as a function of the parameters.

Lookup Consistency By the lookup protocol, a lookup is inconsistent if the immediate predecessor of the sought key has a wrong $s_{1}$ pointer. However, we need only consider the case when the $s_{1}$ pointer is pointing to an alive (but incorrect) node since our implementation of the protocol always requires the lookup to return an alive node as an answer to the query. The probability that a lookup is inconsistent $I(r, \alpha)$ is hence $w_{1}(r, \alpha)-d_{1}(r, \alpha)$. This prediction matches the simulation results very well, as shown in Fig. 10. 


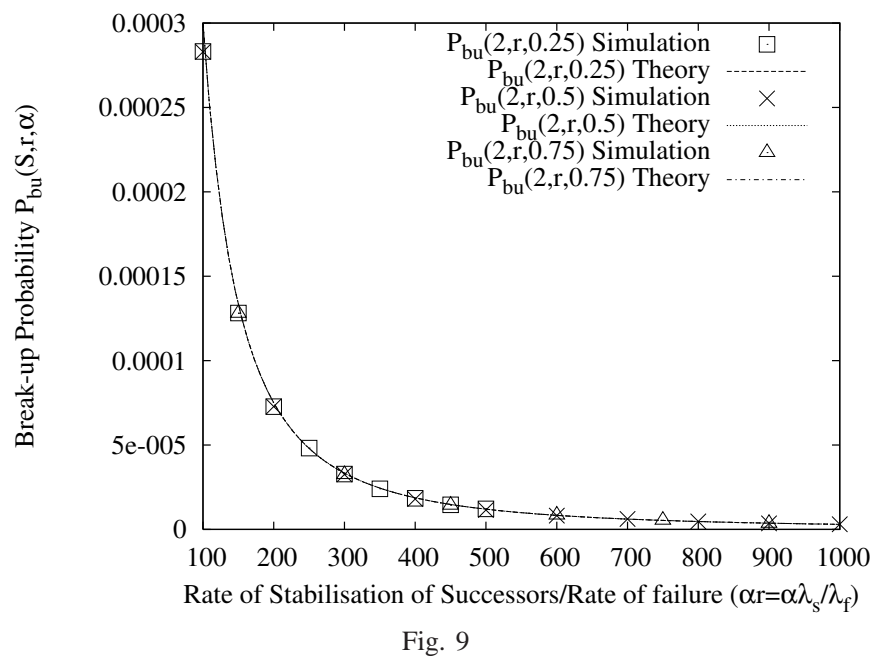

THEORY AND SIMULATION FOR THE BREAK-UP PROBABILITY

$$
P_{b u}(2, r, \alpha) \text {. }
$$

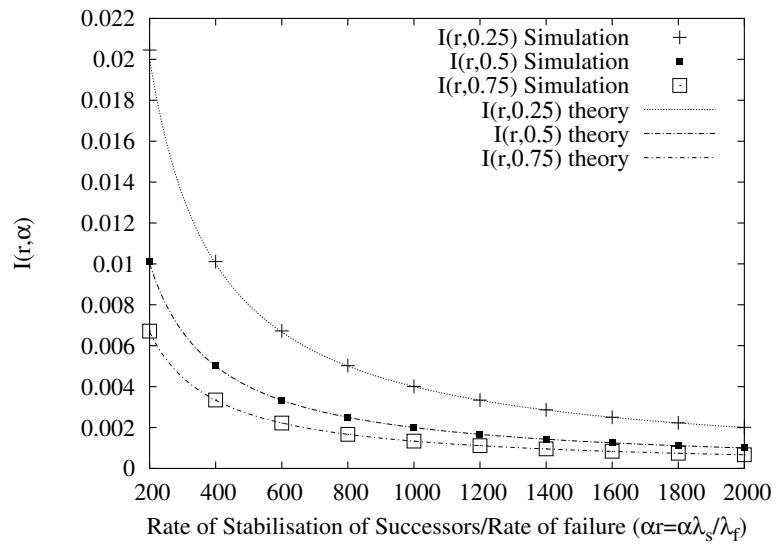

Fig. 10

THEORY AND SIMULATION FOR INCONSISTENT LOOKUPS $I(r, \alpha)$.

\section{Failure of Fingers}

We now turn to estimating the fraction of finger pointers which point to failed nodes. As we will see this is an important quantity for predicting lookups, since failed fingers cause timeouts and increase the lookup length. However, we only need to consider fingers pointing to dead nodes. Unlike members of the successor list, alive fingers even if outdated, always bring a query closer to the destination and do not affect consistency or substantially even the lookup length. Therefore we consider fingers in only two states, alive or dead (failed). By our implementation of the stabilization protocol (see Sections III-A and III-B), fingers and successors are stabilized entirely independently of each other to simplify the analysis. Thus even though the first finger is also always the first successor, this information is not used by the node in updating the finger. Fingers of nodes far apart are independent of each other. Fingers of adjacent nodes can be correlated and we take this into account. The only assumption in this section is in connection with the join protocol as explained below.

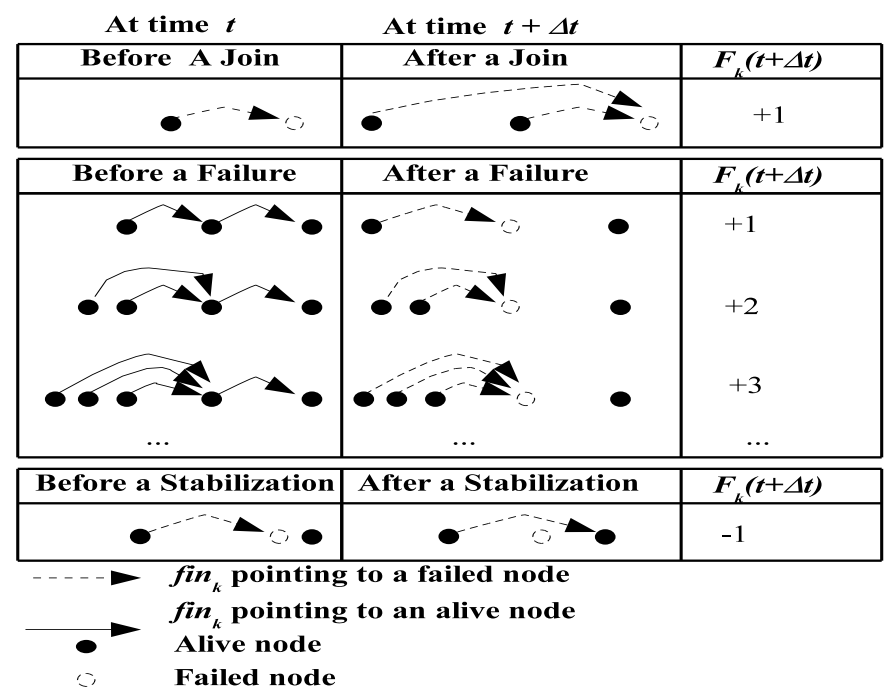

Fig. 11

ChANGes in $F_{k}$, THE NUMBer OF FAILED $f i n_{k}$ POINTERs, DUE to JOINS, FAILURES AND STABILIZATIONS.

TABLE III

THE RELEVANT GAIN AND LOSS TERMS FOR $F_{k}$, THE NUMBER OF NODES WHOSE $k t h$ FINGERS ARE POINTING TO A FAILED NODE FOR $k>1$.

\begin{tabular}{|l|l|}
\hline$F_{k}(t+\Delta t)$ & Probability of Occurence \\
$=F_{k}(t)+1$ & $c_{3.1}=\left(\lambda_{j} N \Delta t\right) \sum_{i=1}^{k} p_{\text {join }}(i, k) f_{i}$ \\
$=F_{k}(t)-1$ & $c_{3.2}=(1-\alpha) \frac{1}{M} f_{k}\left(\lambda_{s} N \Delta t\right)$ \\
$=F_{k}(t)+1$ & $c_{3.3}=\left(1-f_{k}\right)^{2}\left[1-p_{1}(k)\right]\left(\lambda_{f} N \Delta t\right)$ \\
$=F_{k}(t)+2$ & $c_{3.4}=\left(1-f_{k}\right)^{2}\left(p_{1}(k)-p_{2}(k)\right)\left(\lambda_{f} N \Delta t\right)$ \\
$=F_{k}(t)+3$ & $c_{3.5}=\left(1-f_{k}\right)^{2}\left(p_{2}(k)-p_{3}(k)\right)\left(\lambda_{f} N \Delta t\right)$ \\
$=F_{k}(t)$ & $1-\left(c_{3.1}+c_{3.2}+c_{3.3}+c_{3.4}+c_{3.5}\right)$ \\
\hline
\end{tabular}

Let $f_{k}(r, \alpha)$ denote the fraction of nodes whose $k^{t h}$ finger points to a failed node and $F_{k}(r, \alpha)$ denote the respective number. For notational simplicity, we write these as simply $F_{k}$ and $f_{k}$. We can predict this function for any $k$ by again estimating the gain and loss terms for this quantity, caused by a join, failure or stabilization event, and keeping only the most relevant terms. These are listed in Table [III and illustrated in Fig. 11

A join event can play a role here by increasing the number of $F_{k}$ pointers if the successor of the joinee had a failed $i^{t h}$ pointer (occurs with probability $f_{i}$ ) and the joinee replicated this from the successor as the joinee's $k^{\text {th }}$ pointer. (occurs with probability $p_{\text {join }}(i, k)$ from property 4.4). For large enough $k$, this probability is one only for $p_{\text {join }}(k, k)$, that is, the new joinee mostly only replicates the successor's $k$ th pointer as its own $k^{t h}$ pointer. This is what we consider here.

A stabilization evicts a failed pointer if there was one to begin with. The stabilization rate is divided by $\mathcal{M}$, since a node stabilizes any one finger randomly, every time it decides to stabilize a finger at rate $(1-\alpha) \lambda_{s}$.

Given a node $n$ with an alive $k^{\text {th }}$ finger (occurs with probability $1-f_{k}$ ), when the node pointed to by that finger fails, the number of failed $k^{t h}$ fingers $\left(F_{k}\right)$ increases. The amount of this increase depends on the number of immediate predecessors of $n$ that were pointing to the failed node with 
their $k^{t h}$ finger. That number of predecessors could be 0,1 , $2, .$. etc. Using property 4.3 the respective probabilities of those cases are: $1-p_{1}(k), p_{1}(k)-p_{2}(k), p_{2}(k)-p_{3}(k), \ldots$ etc.

Solving for $f_{k}$ in the steady state, we get:

$$
\begin{aligned}
f_{k} & =\frac{\left[2 \tilde{P}_{r e p}(k)+2-p_{\text {join }}(k)+\frac{r(1-\alpha)}{\mathcal{M}}\right]}{2\left(1+\tilde{P}_{r e p}(k)\right)} \\
- & \frac{\sqrt{\left[2 \tilde{P}_{r e p}(k)+2-p_{j o i n}(k)+\frac{r(1-\alpha)}{\mathcal{M}}\right]^{2}-4\left(1+\tilde{P}_{r e p}(k)\right)^{2}}}{2\left(1+\tilde{P}_{r e p}(k)\right)}
\end{aligned}
$$

where $\tilde{P}_{r e p}(k)=\Sigma p_{i}(k)$. In practice, it is enough to keep the first three terms in this sum. To first order in $\frac{1}{r}$ we have, in analogy to (6),

$$
f_{k} \approx \frac{\left(1+\tilde{P}_{r e p}(k)\right) \mathcal{M}}{(1-\alpha) r}
$$

This expression simply says that the fraction of dead fingers is inversely proportional to the rate of finger stabilizations, $(1-\alpha) r$, and proportional to how many fingers there are to stabilize, $\mathcal{M}$, with the proportionality factor $\left(1+\tilde{P}_{\text {rep }}(k)\right)$ depending only on $\rho$.

To sum up, the computation of the fraction of dead $k^{t h}$ finger pointers is analogous to the calculation of the fraction of wrong first successor pointer, albeit a bit more involved. No recursion is involved, in contrast to the calculation of the fraction of wrong higher successor pointers. The above expressions, 10 match very well with the simulation results (Fig. 13).

\section{E. Cost of Finger Stabilizations and Lookups}

In this section, we demonstrate how the information about the failed fingers and successors can be used to predict the cost of stabilizations, lookups or in general the cost for reaching any key in the id space. By cost we mean the number of hops needed to reach the destination including the number of timeouts encountered en-route. Timeouts occur every time a query is passed to a dead node. The node does not answer and the originator of the query has to use another finger instead. For this analysis, we consider timeouts and hops to add equally to the cost. We can easily generalize this analysis to investigate the case when a timeout costs some factor $\gamma$ times the cost of a hop.

Define $C_{t}(r, \alpha)$ (also denoted by $C_{t}$ ) to be the expected cost for a given node to reach some target key which is $t$ keys away from it (which means reaching the first successor of this key). For example, $C_{1}$ would then be the cost of looking up the adjacent key (1 key away). Since the adjacent key is always stored at the first alive successor, therefore if the first successor is alive (which occurs with probability $1-d_{1}$ ), the cost will be 1 hop. If the first successor is dead but the second is alive (occurs with probability $d_{1}\left(1-d_{2}\right)$ ), the cost will be 1 hop +1 timeout $=2$ and the expected cost is $2 \times d_{1}\left(1-d_{2}\right)$ and so forth. Therefore, we have $C_{1}=1-d_{1}+2 \times d_{1}(1-$ $\left.d_{2}\right)+3 \times d_{1} d_{2}\left(1-d_{3}\right)+\cdots \approx 1+d_{1}=1+1 /(\alpha r)$.
To find the expected cost for reaching a general distance $t$ we need to closely follow the Chord protocol, which would lookup $t$ by first finding the closest preceding finger. For the purposes of the analysis, we will find it easier to think in terms of the closest preceding start. Let us hence define $\xi$ to be the start of the finger (say the $k^{t h}$ ) that most closely precedes $t$. Hence $\xi=2^{k-1}+n$ and $t=\xi+m$ i.e., there are $m$ keys between the sought target $t$ and the start of the closest preceding finger. With that, we can write a recursion relation for $C_{\xi+m}$ as follows:

$$
\begin{aligned}
& C_{\xi+m}=C_{\xi}[1-a(m)] \\
& +\left(1-f_{k}\right) a(m)\left[1+\sum_{i=0}^{m-1} b c(i, m) C_{m-i}\right] \\
& +f_{k} a(m)\left[1+\sum_{i=1}^{k-1} h_{k}(i)\right. \\
& \left.\sum_{l=0}^{\xi / 2^{i}-1} b c\left(l, \xi / 2^{i}\right)\left(1+(i-1)+C_{\xi_{i}-l+m}\right)+O\left(h_{k}(k)\right)\right]
\end{aligned}
$$

where $\xi_{i} \equiv \sum_{m=1, i} \xi / 2^{m}$ and $h_{k}(i)$ is the probability that a node is forced to use its $k-i^{t h}$ finger owing to the death of its $k^{t h}$ finger. The probabilities $a, b, b c$ have already been introduced in Section IV and we define the probability $h_{k}(i)$ below.

The lookup equation though rather complicated at first sight merely accounts for all the possibilities that a Chord lookup will encounter, and deals with them exactly as the protocol dictates.

The first term (Fig. 12 (a)) accounts for the eventuality that there is no node intervening between $\xi$ and $\xi+m$ (occurs with probability $1-a(m)$ ). In this case, the cost of looking for $\xi+m$ is the same as the cost for looking for $\xi$.

The second term (Fig. 12 (b)) accounts for the situation when a node does intervene in between (with probability $a(m)$ ), and this node is alive (with probability $1-f_{k}$ ). Then the query is passed on to this node (with 1 added to register the increase in the number of hops) and then the cost depends on the length of the distance between this node and $t$.

The third term (Fig. 12 (c)) accounts for the case when the intervening node is dead (with probability $f_{k}$ ). Then the cost increases by 1 (for a timeout) and the query needs to find an alternative lower finger that most closely precedes the target. Let the $k-i^{t h}$ finger (for some $i, 1 \leq i \leq k-1$ ) be such a finger. This happens with probability $h_{k}(i)$ i.e., the probability that the lookup is passed back to the $k-i^{\text {th }}$ finger either because the intervening fingers are dead or share the same finger table entry as the $k^{t h}$ finger is denoted by $h_{k}(i)$. The start of the $k-i^{t h}$ finger is at $\xi / 2^{i}$ and the distance between $\xi / 2^{i}$ and $\xi$ is equal to $\sum_{m=1, i} \xi / 2^{m}$ which we denote by $\xi_{i}$. Therefore, the distance from the start of the $k-i^{\text {th }}$ to the target is equal to $\xi_{i}+m$. However, note that $\operatorname{fin}_{k-i}$. node could be $l$ keys away (with probability $b c\left(l, \xi / 2^{i}\right)$ ) from fin $_{k-i}$. start (for some $l, 0 \leq l<\xi / 2^{i}$ ). Therefore, after making one hop to fin $_{k-i}$.node, the remaining distance to the target is $\xi_{i}+$ 


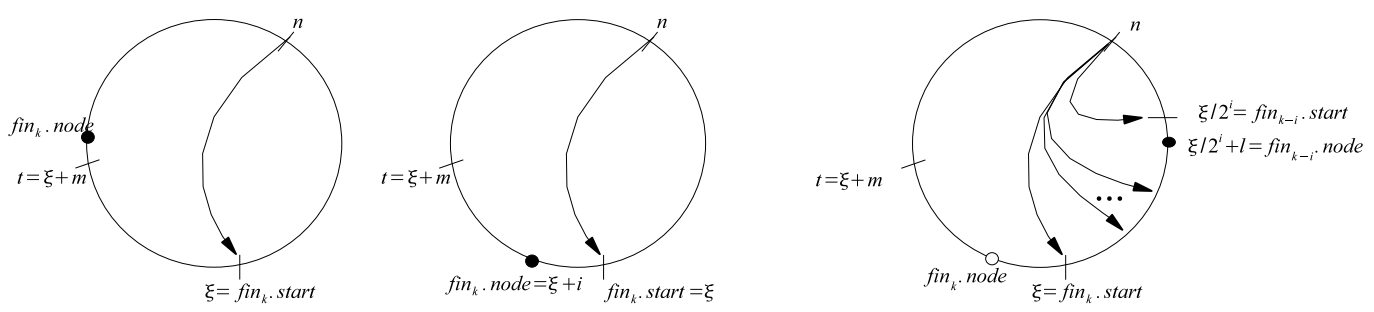

\begin{tabular}{|c|c|l|l|}
\hline Prob. & $1-a(m)$ & \multicolumn{1}{|c|}{$a(m)\left(1-f_{k}\right)$} & $a(m) f_{k} h_{k}(i) b c\left(l, \xi / 2^{i}\right)$ \\
\hline Cost & $C_{\xi+m}=C_{\xi}$ & $\begin{array}{l}C_{\xi+m}=1+b c(i, m) C_{m-i} \\
0 \leq i<m\end{array}$ & $\begin{array}{l}C_{\xi+m}=1+1+C_{\xi_{-}-l+m}+(i-1) \\
\left(C_{t}=C_{\xi+m}\right)\end{array}$ \\
& & $0 \leq i \leq k-1,1 \leq l<\xi / 2^{i}$ \\
\hline
\end{tabular}

(a)

(b)

(c)

Fig. 12

CASES THAT A LOOKUP CAN ENCOUNTER WITH THE RESPECTIVE PROBABILITIES AND COSTS.
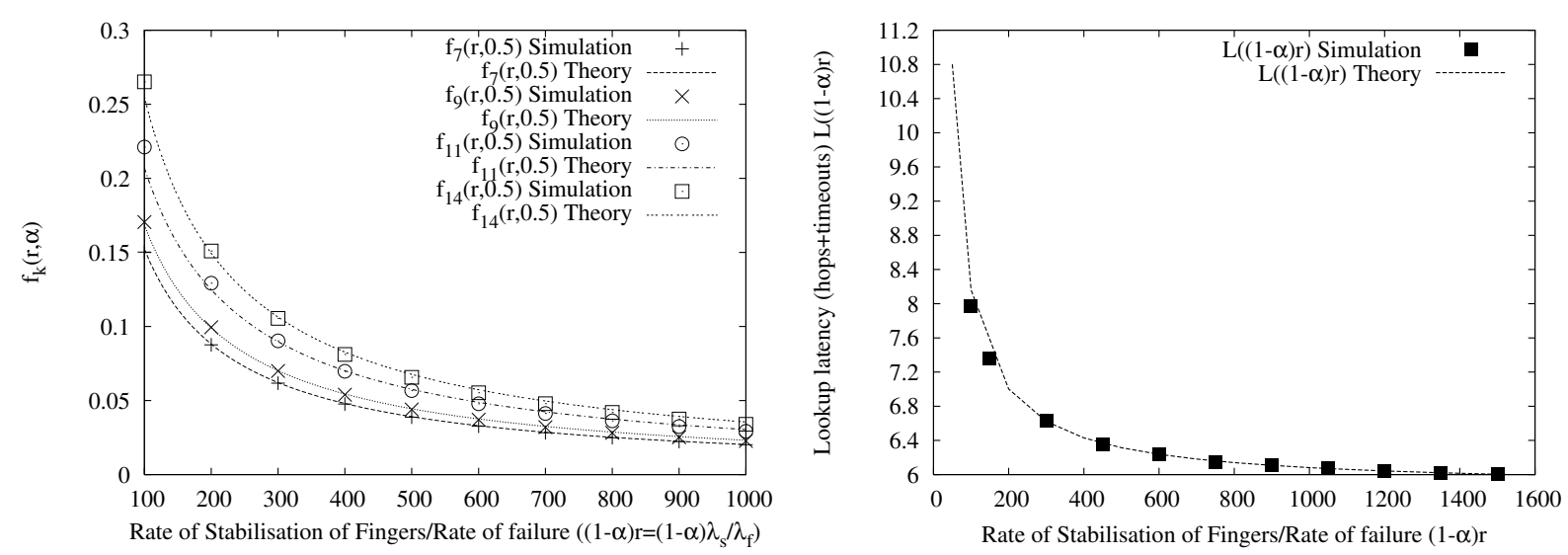

Fig. 13

THEORY AND SIMULATION FOR PROBABILITY OF FAILURE OF THE $k^{\text {th }}$ FINGER $f_{k}(r, \alpha)$, AND THE LOOKUP LENGTH $L(r, \alpha)$.

$m-l$. The increase in cost for this operation is $1+(i-$ 1 ); the 1 indicates the cost of taking up the query again by $f_{i n} n_{k-i}$.node, and the $i-1$ indicates the cost for trying and discarding each of the $i-1$ intervening fingers. The probability $h_{k}(i)$ is easy to compute given property 4.2 and the expression for the $f_{k}$ 's computed in the previous section.

$$
\begin{aligned}
h_{k}(i) & =a\left(\xi / 2^{i}\right)\left(1-f_{k-i}\right) \\
& \times \prod_{s=1, i-1}\left(1-a\left(\xi / 2^{s}\right)+a\left(\xi / 2^{s}\right) f_{k-s}\right), i<k \\
h_{k}(k) & =\prod_{s=1, k-1}\left(1-a\left(\xi / 2^{s}\right)+a\left(\xi / 2^{s}\right) f_{k-s}\right)
\end{aligned}
$$

In (13) we account for all the reasons that a node may have to use its $k-i^{\text {th }}$ finger instead of its $k^{\text {th }}$ finger. This could happen because the intervening fingers were either dead or not distinct. The probabilities $h_{k}(i)$ satisfy the constraint $\sum_{i=1}^{k} h_{k}(i)=1$ since clearly, either a node uses any one of its fingers or it doesn't. This latter probability is $h_{k}(k)$, that is the probability that a node cannot use any earlier entry in its finger table. In this case, $n$ proceeds to its successor list. The query is now passed on to the first alive successor and the new cost is a function of the distance of this node from the target $t$. We indicate this case by the last term in 12 which is $O\left(h_{k}(k)\right)$.
This can again be computed from the inter-node distribution and from the functions $d_{k}(r, \alpha)$ computed earlier. However in practice, the probability for this is extremely small except for targets very close to $n$. Hence this does not significantly affect the value of general lookups and we ignore it in our analysis.

The cost for general lookups is hence

$$
L(r, \alpha)=\frac{\sum_{i=1}^{\mathcal{K}-1} C_{i}(r, \alpha)}{\mathcal{K}}
$$

The lookup equation is solved recursively numerically, given the coefficients and $C_{1}$. In Fig. 13, we compare theoretical results with simulation for $N=1000$. It is seen that the theory matches the simulation results very well.

In Fig. 14 we also show the theoretical predictions for some larger values of $N$. From the structure of Equation 12, it is clear that the dependence of the average lookup on churn comes entirely from the presence of the terms $f_{k}$. Since $f_{k} \sim f$ is independent of $k$ for large fingers, we can approximate the average lookup length by the functional form $L(r, \alpha)=A+B f+C f^{2}+\cdots$. The coefficients $A, B, C$ etc can be recursively computed by solving the lookup equation to the required order in $f$ and depend only on $N$ the number of 




Fig. 14

LOOKUP COST, THEORETICAL CURVE, FOR 1000,2000,4000,8000 AND 16000 PEERS. THE RATIONALE FOR THE FITS IS EXPLAINED IN THE TEXT.

nodes, $1-\rho$ the density of peers and $b$ the base or equivalently the size of the finger table of each node. The advantage of writing the lookup length this way is that churn-specific details such as how new joinees construct a finger table or how exactly stabilizations are done in the system, can be isolated in the expression for $f$. If we were to change our stabilization strategy for example [9], we could immediately estimate the lookup length by plugging in the new expression for $f$ in the above relation.

The coefficient $A$, which is the lookup cost without churn can be obtained very precisely for any base $b$, from analyzing (12) in the zero-churn case. This analysis is rather laborious and will be presented elsewhere [9]. It confirms the wellknown result $A=\frac{1}{2} \log _{2} N$ and in addition reproduces small deviations from this behavior previously observed by us in numerical simulations [7]. The values of $A$ in Fig. 14 are taken from this analysis.

$B$ can be qualitatively estimated as follows : every sufficiently long finger is dead with some finite probability $f$ given by 10 . If $A$ is the average value of the lookup length without churn, then each look-up encounters $f A$ dead fingers on average. This estimate predicts a look-up cost of approximately $A(1+f)$, giving $B=A$ and $C$ and all other coefficients equal to 0 ..

In Fig. 14 we show that the best fit to the data is obtained in fact by taking $B=A$ and $C=3 A$. The expression for $f$ is taken from 10 for large $k$ (for a system with 20 fingers, the expression for $f_{k}$ becomes independent of $k$ for $k \geq 13)$. In general, as mentioned earlier, $B$ and $C$ can be obtained accurately for any value of the system parameters by the numerical solution of Eq. 12 to the required order.

\section{Discussion AND CONCLUSION}

In this paper we have presented a detailed theoretical analysis of a DHT-based P2P system, Chord, using a fluid model. The technique for deriving the fluid model has been borrowed from the master equation approach of physics, which helps in systematically taking different dynamical effects into account. This analysis differs from previous theoretical work done on DHTs in that it aims not at establishing bounds, but on precise determination of the relevant quantities in this dynamically evolving system. From the match of our theory and the simulations, it can be seen that we can predict with an accuracy of greater than $1 \%$ in most cases. Though this analysis is not exact, since it takes only some (and not all) correlations into account, yet it provides a methodology for keeping track of most of the relevant details of the system. We expect that a similar analysis can be done for most other DHT's, thus helping to establish quantitative guidelines for their comparison.

The main conclusions for the analysis of Chord in a statistically steady state are the following.

Property 5.1: As a function of $r$, the ratio of the rate of stabilizations to the rate of failures, the fraction of wrong pointers of any kind (successors or fingers) is to leading order and good approximation Const. $/ r$, where the constant depends on the pointer.

Property 5.2: The probability of break up of a ring can be estimated from the knowledge of the fraction of wrong first successors, wrong second successors, etc. This probability is generally very low when every node has a sufficient number of successors, indicating that Chord is robust against ring breakup.

Property 5.3: At a given value of $r$, the fraction of wrong successors, $w_{k}$, and the fraction of dead fingers, $f_{k}$, increases with $k$. The fraction of wrong successors increases indefinitely, and becomes of order one at $k$ about $\sqrt{r}$ for the particular stabilization strategy that we have used. The fraction of dead fingers on the other hand tends to a constant for sufficiently large $k$.

Property 5.4: The look-up cost, which is the expected number of hops including time-outs, can be computed by numerical recursion. The fraction of incorrect finger pointers $f_{k}(\sim f$ for large $k$ ) is a required input for this recursion. The lookup cost tends to the well-known average number of hops without churn when $f$ is small (or churn is low) and increases when $f$ is large. We show that it can be well described by the formula $A(1+g(f))$, where $A$ is the value of the lookup cost without churn and $g(f)$ is well approximated by $f+3 f^{2}$ for $N<<K$. In general $g(f)$ can be obtained accurately to any desired order by solving Eq. 12 recursively to the required order in $f$.

Property 5.5: The preceding note brings out the following simple feature of Chord: under any state of churn, sufficiently long fingers are all dead with essentially the same probability. Hence, in a sufficiently large system, a look-up will almost surely encounter one or more dead fingers, leading to timeouts. For applications where time-outs should be the exception and not the norm, this paper helps in estimating how much stabilization is necessary under a given level of churn, to achieve such a level of performance.

Property 5.6: The preceding note also brings out the additional feature that by writing the lookup cost in the above simplified form, we can isolate the effects of churn-specific details in the expression for $f$. Changing details in the join protocol or changing the maintenance strategy [9] merely cause a change in the expression for $f$. The lookup cost with this new strategy can then be immediately assessed for any $r$, 
by plugging in the new expression for $f$ in the expression for the lookup cost (as opposed to solving Eq. 12 each time for each value of $r$ ).

The impact of this work can be summarized as follows: given that periodic stabilization is a fundamental technique for topology maintenance in DHTs, the question: "How often should a DHT node perform periodic stabilization?" is of great practical relevance. The answer to this question depends on several factors. First we need to know where the DHT is deployed, in a LAN, in a cooperative milieu, or among public non-trusting partners, i.e., what is the expected join/failure rate (churn)? Secondly, since DHTs involve different types of stabilizations, we need to know which of these rates is of interest to optimize. For example, in the DHT studied in this paper, there is both ring stabilization as well as finger stabilization. Thirdly, we also need to know whether we have performance goals which require us to know how much stabilization is needed, or constraints on bandwidth which necessitate a knowledge of the expected performance. Previous analytical attempts (see Section III) have addressed these question through the identification of general (algorithm/system-neutral) bounds on stabilization rates.

In this paper, we have taken another point of view. We have traded-off generality for accuracy. That is, we have produced results that can describe to a very high degree of accuracy quantities like the probability of inconsistent look-ups and the expected look-up length as functions of the stabilization and churn rates. Many of the insights we get from this analysis such as most of the points listed above, would be very hard to come by from simulations alone. So for instance, the formulae produced in this paper could directly be used by a system administrator or the person in charge of deploying a DHT as a guide for configuring stabilization rates. While the results are based on Chord, all analyses concerning the ring (breakup and inconsistency) are applicable to many other systems, since consistent hashing on a ring is a recurring component in many other DHTs.

\section{LimitATIONS AND FUTURE WORK}

The main limitation of this work stems from the fact that the results are inherently dependent on the intricate details of the analyzed algorithms. While some changes in the algorithms can be easily accommodated without redoing the analysis (as explained in 5.6, others such as a different lookup strategy or a different placement of fingers would necessitate recalculating all the quantities again. However, results concerning the ringrelated aspects like successor lists, break-up probability and inter-node distributions are likely to be reusable in other variations of the Chord protocols as well other systems using a ring geometry.

For the future, the authors' research agenda include the introduction of extensions to the current model to be able to account for locality-awareness and different topology maintenance techniques. Some work towards the latter goal has already been done in [9]. Relatedly, a useful application for this work is to enable systems to dynamically self-tune their stabilization rates and choose the best maintenance technique to achieve a desired hop count.

\section{REFERENCES}

[1] Karl Aberer, Anwitaman Datta, and Manfred Hauswirth, Efficient, selfcontained handling of identity in peer-to-peer systems, IEEE Transactions on Knowledge and Data Engineering 16 (2004), no. 7, 858-869.

[2] D. Anick, D. Mitra, and M.M. Sondhi, Stochastic theory of datahandling systems with multiple sources, Bell Systems Technical Journal 61 (1982), 1871-1894.

[3] James Aspnes, Zoë Diamadi, and Gauri Shah, Fault-tolerant routing in peer-to-peer systems, Proceedings of the twenty-first annual symposium on Principles of distributed computing, ACM Press, 2002, pp. 223-232.

[4] E. Brockmeyer, H.L. Halstrom, and Arns Jensen, The life and works of A.K. Erlang, The Copenhagen Telephone Company, 1948.

[5] Miguel Castro, Manuel Costa, and Antony Rowstron, Performance and dependability of structured peer-to-peer overlays, Proceedings of the 2004 International Conference on Dependable Systems and Networks (DSN'04), IEEE Computer Society, 2004.

[6] Florence Clévenot and Philippe Nain, A simple fluid model for the analysis of the squirrel peer-to-peer caching system, IEEE INFOCOM 2004, 2004.

[7] Sameh El-Ansary, Erik Aurell, and Seif Haridi, A physics-inspired performace evaluation of a structured peer-to-peer overlay network, The International Conference on Parallel and Distributed Computing and Networks (PDCN 2005), 2005.

[8] Supriya Krishnamurthy, Sameh El-Ansary, Erik Aurell, and Seif Haridi, A statistical theory of chord under churn, The 4th International Workshop on Peer-to-Peer Systems (IPTPS'05) (Ithaca, New York), February 2005.

[9] _ Comparing maintenance strategies for overlays, Tech. report, Swedish Institute of Computer Science, in preparation 2007.

[10] Jinyang Li, Jeremy Stribling, Robert Morris, M. Frans Kaashoek, and Thomer M. Gil, A performance vs. cost framework for evaluating dht design tradeoffs under churn, Proceedings of the 24th Infocom (Miami, FL), March 2005

[11] David Liben-Nowell, Hari Balakrishnan, and David Karger, Analysis of the evolution of peer-to-peer systems, ACM Conf. on Principles of Distributed Computing (PODC) (Monterey, CA), July 2002.

[12] N.G. van Kampen, Stochastic Processes in Physics and Chemistry, North-Holland Publishing Company, 1981, ISBN-0-444-86200-5.

[13] Dongyu Qui and R. Srikant, Modeling and performance analysis of bittorrent-like peer-to-peer networks, SIGCOMM'04 (Portland, Oregon), August 2004.

[14] Sean Rhea, Dennis Geels, Timothy Roscoe, and John Kubiatowicz, Handling churn in a DHT, Proceedings of the 2004 USENIX Annual Technical Conference(USENIX '04) (Boston, Massachusetts, USA), June 2004.

[15] Ion Stoica, Robert Morris, David Liben-Nowell, David Karger, M. Frans Kaashoek, Frank Dabek, and Hari Balakrishnan, Chord: A scalable peerto-peer lookup service for internet applications, IEEE Transactions on Networking 11 (2003).

[16] Shengquan Wang, Dong Xuan, and Wei Zhao, On resilience of structured peer-to-peer systems, GLOBECOM 2003 - IEEE Global Telecommunications Conference, Dec 2003, pp. 3851-3856. 